\title{
Hybrid Heuristic Algorithm for GPS Surveying Problem
}

\author{
Stefka Fidanova \\ IPP - BAS, Acad. G. Bonchev str. bl.25A, 1113 Sofia, Bulgaria \\ stefka@parallel.bas.bg
}

\begin{abstract}
This paper introduces several approaches based on ant colony optimization for efficient scheduling the surveying activities of designing satellite surveying networks. These proposed approaches use a set of agents called ants that cooperate to iteratively construct potential observation schedules. Within the context of satellite surveying, a positioning network can be defined as a set of points which are coordinated by placing receivers on these point to determine sessions between them. The problem is to search for the best order in which these sessions can be observed to give the best possible schedule. The same problem arise in Mobile Phone Surveying networks. Several case studies have been used to experimentally assess the performance of the proposed approaches in terms of solution quality and computational effort.
\end{abstract}

\section{Introduction}

The continuing research on naturally occurring social systems offers the prospect of creating artificial systems that generate practical solutions to many Combinatorial Optimization Problems (COPs). Metaheuristic techniques have evolved rapidly in an attempt to find good solutions to these problems within a desired time frame [6]. They attempt to solve complex optimization problems by incorporating processes which are observed at work in real life [2]3. For example, in the case of ants, using their simple individual interactions mediated by pheromone, they can collectively determine the shortest route from their nest to a food source without using visual cues [1. When applied to satellite surveying, these techniques can assist surveyors in creating a better observation schedule for designing the whole positioning network.

The purpose of surveying is to determine the locations of points on the earth. Measuring tapes or chains require that the survey crew physically pass through all the intervening terrain to measure the distance between two points. Surveying methods have undergone a revolutionary change over that last few years with the deployment of the satellite navigation systems. The most widely known space systems are: the American Global Positioning System (GPS), the Russian GLObal Navigation Satellite System (GLONASS), and the forthcoming European Satellite Navigation System (GALILEO). In this paper, it is the use of GPS to establish surveying networks that is being investigated. GPS satellites continuously transmit electrical signals to the earth while orbiting the earth. A receiver,

T. Boyanov et al. (Eds.): NMA 2006, LNCS 4310, pp. 239246 2007.

(C) Springer-Verlag Berlin Heidelberg 2007 
with unknown position on the earth, has to detect and convert the signals transmitted from all of the satellites into useful measurements. These measurements would allow a user to compute a three-dimensional coordinates position for the location of the receiver. To maximize the benefit of using this technique, several procedures based on Ant Colony Optimization, have been developed and implemented to find an efficient schedule to improve the performance and explore the search space more effectively 8 . In this paper we implement an Ant Colony Optimization (ACO) algorithm applied to GPS surveying networks and the general case of the problem is addressed by presenting several case studies and the obtained numerical results. Our aim is to suggest various local search procedures, to combine them with ACO technique and to check which one is the best for this problem.

The rest of this paper is organized as follows. The general framework for GPS surveying network problem as a combinatorial optimization problem is described in Section 2. Then, the search strategy of the ACO is explained in Section 3. Various local search procedures are described in Section 4. The ACO algorithm coupled with local search procedures, applied to GPS surveying networks, is outlined and the general case of the problem is addressed by presenting several case studies and the obtained numerical results in Section 5. The paper ends with a summary of the conclusions and directions for future research.

\section{Formulating the GPS Surveying Network Problem}

A GPS network is distinctly different from a classical survey network in that no inter-visibility between stations is required. In GPS surveying, after defining the locations of the points for an area to be surveyed, GPS receivers will be used to map this area by creating a network of these coordinated points. These points, control stations within the context of surveying, are fixed on the ground and located by an experienced surveyor according to the nature of the land and the requirements of the survey [5]. At least two receivers are required to simultaneously observe GPS satellites, for a fixed period of time, where each receiver is mounted on each station. The immediate outcome of the observation is a session between these two stations. After completing the first stage of sessions observation and defining the locations of the surveyed stations, the receivers are moved to other stations for similar tasks till the whole network is completely observed according to an observation schedule. The total cost of carrying out the above survey, which is computed upon the criteria to be minimized, represents the cost of moving receivers between stations. The problem is to search for the best order, with respect to the time, in which these sessions can be observed to give the cheapest schedule $\mathrm{V}$, i.e.:

$$
\text { Minimize: } C(V)=\sum_{p \in R} C\left(S_{p}\right)
$$

where: $C(V)$ is the total cost of a feasible schedule $V(N, R, U)$; 
$S_{p}$ is the route of the receiver $p$ in a schedule;

$N$ is the set of stations $N=1, \ldots, n$;

$R$ is the set of receivers $R=1, \ldots, r$;

$U$ is the set of sessions $U=1, \ldots, u$.

\section{Ant Colony Optimization}

Real ants foraging for food lay down quantities of pheromone (chemical cues) marking the path that they follow. An isolated ant moves essentially at random but an ant encountering a previously laid pheromone will detect it and decide to follow it with high probability and thereby reinforce it with a further quantity of pheromone. The repetition of the above mechanism represents the auto-catalytic behavior of real ant colony where the more the ants follow a trail, the more attractive that trail becomes.

The ACO algorithm uses a colony of artificial ants that behave as cooperative agents in a mathematics space were they are allowed to search and reinforce pathways (solutions) in order to find the optimal ones. The problem is represented by graph and the ants walk on the graph to construct solutions. After initialization of the pheromone trails, ants construct feasible solutions, starting from random nodes, then the pheromone trails are updated. At each step ants compute a set of feasible moves and select the best one (according to some probabilistic rules) to carry out the rest of the tour. The transition probability is based on the heuristic information and pheromone trail level of the move. The higher value of the pheromone and the heuristic information, the more profitable is to select this move and resume the search. In the beginning, the initial pheromone level is set to a small positive constant value $\tau_{0}$ and then ants update this value after completing the construction stage. ACO algorithms adopt different criteria to update the pheromone level. In our implementation we use Ant Colony System (ACS) 4] approach.

In ACS the pheromone updating consists of two stages: local update stage and global update stage.

\subsection{Local Update Stage}

While ants build their solution, at the same time they locally update the pheromone level of the visited paths by applying the local update rule as follows:

$$
\tau_{i j} \leftarrow(1-\rho) \tau_{i j}+\rho \tau_{0}
$$

Where $\tau_{i j}$ is an amount of the pheromone on the arc $(i, j)$ of the graph of the problem, $\rho$ is a persistence of the trail and term $(1-\rho)$ can be interpreted as trail evaporation.

The aim of the local updating rule is to make better use of the pheromone information by dynamically changing the desirability of edges. Using this rule, ants will search in a wide neighborhood of the best previous solution. As shown in the formula, the pheromone level on the paths is highly related to the value 
of evaporation parameter $\rho$. The pheromone level will be reduced and this will reduce the chance that the other ants will select the same solution and consequently the search will be more diversified.

\subsection{Global Updating Stage}

When all ants have completed their solution, the pheromone level is updated by applying the global updating rule only on the paths that belong to the best solution since the beginning of the trial as follows:

$$
\tau_{i j} \leftarrow(1-\rho) \tau_{i j}+\Delta \tau_{i j}
$$

where $\Delta \tau_{i j}= \begin{cases}1 / C_{g b} & \text { if }(i, j) \in \text { best solution } \\ 0 & \text { otherwise }\end{cases}$

$C_{g b}$ is the cost of the best solution from the beginning. This global updating rule is intended to provide a greater amount of pheromone on the paths of the best solution, thus intensify the search around this solution. The transition probability to select the next node is given as:

$$
\operatorname{prob}_{i j}^{k}(t)= \begin{cases}\frac{\tau_{i j}^{\alpha} \eta_{i j}^{\beta}}{\sum_{\text {seallowed }_{k}(t)} \tau_{i s}^{\alpha} \eta_{i s}^{\beta}} & \text { if } j \in \text { allowed }_{k}(t) \\ 0 & \text { otherwise }\end{cases}
$$

where $\tau_{i j}$ is the intensity measure of the pheromone deposited by each ant on the path $(i, j), \alpha$ the intensity control parameter, $\eta_{i j}$ is the visibility measure of the quality of the path $(i, j)$. This visibility is determined by $\eta_{i j}=1 / l_{i j}$, where $l_{i j}$ is the cost of move from session $i$ to session $j . \beta$ is the visibility parameter and allowed $_{k}(t)$ is the set of remaining feasible sessions. Thus the higher the value of $\tau_{i j}$ and $\eta_{i j}$, the more profitable it is to include item $j$ in the partial solution.

\section{Local Search Strategy}

The Local Search (LS) method (move-by-move method) perturbs a given solution to generate different neighborhoods using a move generation mechanism. LS attempts to improve an initial solution by a series of local improving changes. A move-generation is a transition from a solution $S$ to another one $S^{\prime} \in V(S)$ in one step. These solutions are selected and accepted according to some pre-defined criteria. The returned solution $S^{\prime}$ may not be optimal, but it is the best solution in its local neighborhood $V(S)$. A local optimal solution is a solution with the local minimal possible cost value. Knowledge of a solution space is the essential key to more efficient search strategies. These strategies are designed to use this prior knowledge and to overcome the complexity of an exhaustive search by organizing searches across the alternatives offered by a particular representation 

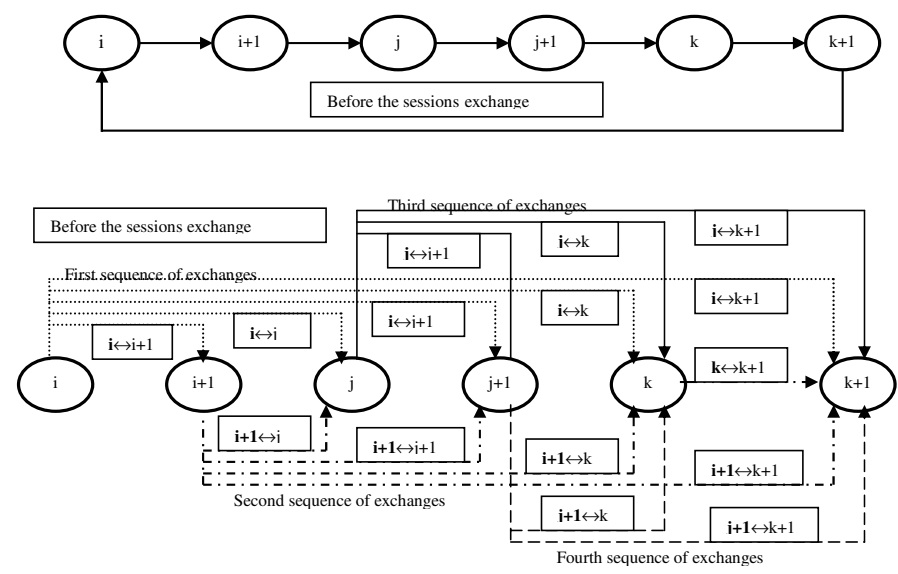

Fig. 1. The sequential local search structure (a)

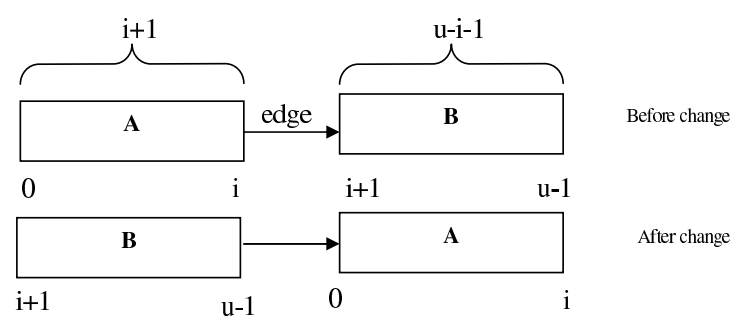

Fig. 2. Local search structure (b1) with 1-exchange of edges

of the solution. The main purpose of LS implementation is to speed up and improve the solutions constructed by the ACO.

In this paper, various local search structures that satisfy the requirements of the GPS network, have been developed and implemented to search the schedule space more effectively. The problem is represented by graph. The nodes correspond to the sessions and the edges represents the distance. A solution is a path in a graph (chain of nodes) which contains all nodes.

The first procedure 8 89, shown in a Figure 1, is based on the sequential session-interchange, the potential pair-swaps are examined in the order $\{1,2\}$, $\{1,3\}, \ldots,\{1, u\},\{2,3\},\{2,4\},\{u-1, u\}$ (For comparison reasons this sequential local search procedure is called structure a).

In the second procedure as shown graphically in Figure 2, $A$ and $B$ are chains of nodes (solution slices), while $\{0, i\}$ and $\{i+1, u\}$ are the first and the last nodes of the chains $A$ and $B$ respectively. In this procedure, only one exchange has been performed of new edge $(u-1,0)$ each iteration and this can be done by selecting one edge $(i, i+1)$ to be removed (For comparison reasons this local search procedure is called structure b1).

In the third procedure as shown graphically in Figure 3, $A, B$ and $C$ are chains of nodes (part of the solution), while $\{0, i\},\{i+1, j\}$, and $\{j+1, u-1\}$ are the 

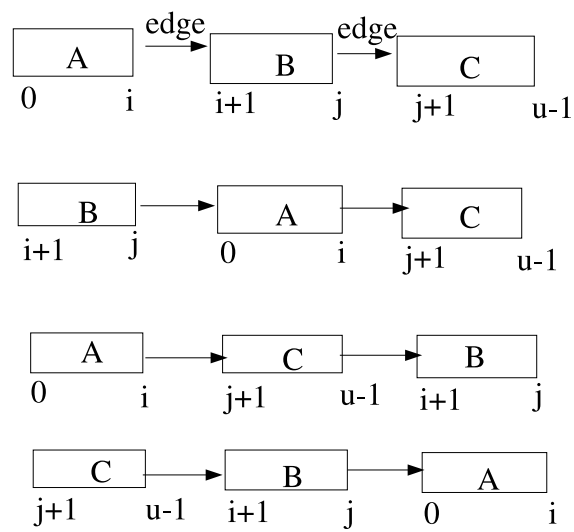

Fig. 3. Local search structure (b2) with 2-exchange of edges

first and the last nodes of the chains $A, B$ and $C$ respectively. In the case of a 2-exchange, there are several possibilities to build a new solution where the edges $(i, i+1)$ and $(j, j+1)$ are selected to be removed in one iteration (For comparison reasons this local search procedure is called structure b2). The possibilities for new edges are $(j, 0)$ and $(i, j+1),(i, j+1)$ and $(u-1, i+1),(u-1, i+1)$ and $(j, 0)$ where $0 \leq i<j \leq u-1$. In both procedures $u$ is the number of sessions in the observed network.

\section{$5 \quad$ Experimental Results}

This section reports on the computational experience of the ACS coupled with various local search procedures using real GPS networks. The first network is a GPS network for Malta and consists of 38 sessions [8]. The initial schedule with a cost of 1405 minutes was composed. The second network is a GPS network for the Seychelles and consists of 71 sessions 9 . The initial schedule with a cost of 994 minutes was composed. The performance of the developed metaheuristic techniques were evaluated with respect to the schedule quality and computational effort using the following measure:

$$
R R C=\left[\left(C_{I N T}-C(V)\right) / C_{I N T}\right] * 100
$$

Where:

$R R C$ is the Relative Reduction of the Cost;

$C_{I N T}$ is the Cost of the Initial Schedule $V_{I N T}$ obtained randomly.

$C_{a}$ is the Cost of the metaheuristic schedule obtained by procedure $a$.

$C_{b 1}$ is the Cost of the metaheuristic schedule obtained by procedure $b 1$.

$C_{b 2}$ is the Cost of the metaheuristic schedule obtained by procedure $b 2$.

$T S$ is the Cost of the metaheuristic schedule in [89] obtained by tabu search.

$S A$ is the Cost of the metaheuristic schedule in [8] obtained by simulated annealing. 
Table 1. Comparison of local search techniques applied to different types of GPS networks

\begin{tabular}{|l|r|r|r|r|}
\hline Data & $\begin{array}{r}\text { Malta } \\
\text { network }\end{array}$ & $R R C$ & $\begin{array}{r}\text { Seychelles } \\
\text { network }\end{array}$ & $R R C$ \\
\hline$U$ & 38 & & 71 & \\
\hline$C_{I N T}$ & 1405 & 0 & 994 & 0 \\
\hline$C_{a}$ & 880 & 37.37 & 859 & 13.58 \\
\hline$C_{b 1}$ & 685 & 51.24 & 807 & 18.81 \\
\hline$C_{b 2}$ & 850 & 39.50 & 871 & 12.37 \\
\hline$T S$ & 1075 & 23.5 & 933 & 6.14 \\
\hline$S A$ & 1375 & 2.14 & 969 & 2.52 \\
\hline
\end{tabular}

The following parameter settings is used: $\rho=0.4, \alpha=\beta=1$. The initial amount of the pheromone was set to a fixed value $\tau_{0}=0.0005$ on all edges, while the number of iterations was set to 200 . The reported results are average results over 20 runs. The developed technique has been coded in $\mathrm{C}++$ and implemented on a Pentium 3 with $900 \mathrm{MHz}$ speed and $512 \mathrm{MB}$ RAM.

The Table 1 shows the advantage of ACO techniques using various local search procedures. ACO technique using local search procedure (b1) produced the best results. With respect to the Malta network with size of 38 sessions, the RRC of the metaheuristic schedule produced by the ACO technique is $37.37 \%$ using local search procedure (a), compared to RRC of $51.24 \%$ using local search procedure (b1), RRC of $39.50 \%$ using local search procedure (b2). In the Seychelles network with size of 71 sessions, the RRC of the metaheuristic schedule produced by the ACO is $13.58 \%$ using local search procedure (a), compared to RRC of $18.81 \%$ using local search procedure (b1), RRC of $12.37 \%$ using local search procedure (b2). In this comparison, local search procedure (b1) gives better results than the other local search procedures for the both GPS networks. ACO is a constructive method and the selected set of neighbors has not so important role in achieving good results. Having better results using the procedure (b1) is due its features: more economic and less perturbs the pheromone. All ACO algorithm outperforms TS and SA.

\section{Concluding Remarks and Future Work}

In this paper, two local search procedures have been developed and compared with local search procedure from [8] and 9] (structure a). The comparison of the performance of the ACO with these procedures applied to different types and sizes of GPS networks is reported. The obtained results are encouraging and the ability of the developed techniques to generate rapidly high-quality solutions for 
observing GPS networks can be seen. For the future work, the developed local search procedures in this paper will be coupled with other metaheuristics to search for further improvement and provide a practical comparison, based on the success of the developed ACO algorithm for constructing optimal solutions for large GPS positioning networks which form the basic framework for geomatic information that support all the environmental activities in the countries.

Acknowledgments. The author is supported by European community grant BIS $21++$.

\section{References}

1. Beckers R., Deneubourg J.L., Gross S. (1992), Trail and U-turns in the Selection of the Shortest Path by the Ants, J. of Theoretical Biology 159,397-415.

2. Bonabeau E., Dorigo M., Theraulaz G. (1999),Swarm Intelligence: From Natural to Artificial Systems, Oxford University Press, New York.

3. Corne D., Dorigo M., Glover F. eds.(1999), New Ideas in Optimization, McCraw Hill, London.

4. Dorigo M., Gambardella L.M. (1997), Ant Colony System: A Cooperative Learning Approach to the Traveling Salesman Problem, IEEE Transactions on Evolutionary Computation 1, 53-66.

5. Leick, A. (1995): GPS Satellite Surveying, 2nd. ed.. Wiley, Chichester, England.

6. Osman I.H., Kelly J.P. (1996): Metaheuristics:An overview. In Osman I.H., Kelly J.P. eds. Metaheuristics:Theory and Applications, Kluwer Acad. Publisher.

7. Reeves, C. R. (editor). (1993): Modern Heuristic Techniques for Combinatorial Problems. Blackwell Scientific Publications, Oxford, England.

8. Saleh H. A., and P. Dare,(2001): Effective Heuristics for the GPS Survey Network of Malta: Simulated Annealing and Tabu Search Techniques. Journal of Heuristics N 7 (6), 533-549.

9. Saleh, H. A., and Dare, P. J. (2002): Heuristic methods for designing Global Positioning System Surveying Network in the Republic of Seychelles. The Arabian Journal for Science and Engineering N 26, 73-93.

10. Saleh, H. A. and Dare, P., (2000): Local Search Strategy to produce Schedules for a GPS Surveying Network. In: Eleventh Young Operational Research Conference (YOR11), University of Cambridge, Cambridge, UK, 28-30 March, (editor A. Tuson), 2000-ORS, UK, ISBN 0-903440-202, 87-103. 